\title{
A Modified Closed Cartilage-Preserving Otoplasty Technique for Prominent Ear Correction
}

\author{
Tae Joon Choi, Jin Sik Burm, \\ Yung Ki Lee
}

Department of Plastic Surgery, Kyung Hee University School of Medicine, Seoul, Korea

This article was presented as a free-standing paper at the 2015 International Meeting of the Korean Society for Aesthetic Plastic Surgery on March 28-29, 2015, in Seoul, Korea.

No potential conflict of interest relevant to this article was reported.
Background In the surgical correction of prominent ear, a technique known as percutaneous adjustable closed otoplasty (PACO), which does not involve skin incision, undermining, or cartilage manipulation, has been developed to resolve problems including hematoma, infection, contour deformities, prolonged use of a compressive dressing, and hospitalization. We modified this procedure to make it more practical and accessible and to achieve better results. In this article, we introduce our modifications and demonstrate the clinical applicability of the modified procedure to patients with hardened auricular cartilage.

Methods Two adult patients with prominent upper ears underwent closed otoplasty in an outpatient setting. Based on the anatomical features of the patients, three lines for traction sutures were designed on the scapha and counter scapha. Tab incisions were made at all predetermined puncture sites. Three antihelix-forming sutures (4-0 nylon) were put in place via percutaneous punctures. The sutures were performed from the counter scapha to the postauricular sulcus subcutaneously, using an 18-mm empty curved needle. The sutures were scraped over the mastoid bone such that they were anchored to the mastoid periosteum. After determining an adequate auriculocephalic distance, the sutures were tied at the postauricular sulcus. A slight overcorrection was made to compensate for post-surgical relapse.

Results We observed no complications such as hematoma, infection, contour deformities, epithelial inclusion cyst formation, suture extrusion, or dimples on the scapha. At a long-term follow-up examination, the patients had well-defined antihelical folds and were satisfied with the aesthetic results of the procedure.

Conclusions We propose our technique as a reliable treatment option for the correction of prominent ear.

Keywords Ear auricle, Ear deformities acquired, Otologic surgical procedures, Surgery plastic

\section{INTRODUCTION}

Prominent ear is a relatively common deformity of the external ear, with an incidence of approximately $5 \%$ in Caucasians [1]. The main

Received: Feb 29, 2016 Revised: May 16, 2016 Accepted: May 19, 2016 Correspondence: Jin Sik Burm Department of Plastic Surgery, Kyung Hee University School of Medicine, 23 Kyungheedae-ro, Dongdaemun-gu, Seoul 02447, Korea. E-mail: jsburm@gmail.com

Copyright @ 2016 The Korean Society for Aesthetic Plastic Surgery.

This is an Open Access article distributed under the terms of the Creative Commons Attribution Non-Commercial License (http://creativecommons.org/licenses/by-nc/4.0/) which permits unrestricted non-commercial use, distribution, and reproduction in any medium, provided the original work is properly cited. www.e-aaps.org anatomical features of prominent ear are protrusion of the upper half of the helix with a wide conchoscaphal angle that results from failure of the antihelix to fold properly, in conjunction with a wide conchomastoid angle, deep conchal bowl, or absence of the helical roll [2]. Prominent ear may cause psychological stress, emotional trauma, and behavioral disorders, particularly in children [3]. The anatomical goal of prominent ear correction is to produce a smooth, round, and well-defined antihelical fold, a conchoscaphal angle of $90^{\circ}$, and conchal reduction or reduction of the conchomastoid angle [4]. A range of techniques have been developed to achieve these goals. Otoplastic techniques are divided into three broad categories: cartilage-cutting techniques, cartilage-sparing techniques, and incisionless techniques. Cartilage-sparing techniques have been 
widely used for prominent ear correction [5]. Such techniques require skin incision and undermining in order to score or rasp the cartilage. Therefore, they pose the risk of hematoma and require postoperative drains or a compressive dressing $[5,6]$. In order to resolve these problems, closed otoplasty techniques without skin undermining and cartilage manipulation, such as incisionless otoplasty [7] and percutaneous adjustable closed otoplasty (PACO) [5], have been developed. PACO is highly effective and has several advantages, but we nonetheless sought to modify it in order to make it more practical and accessible and to achieve better results. The suture material and suture carrier traditionally used in PACO are costly and are not readily available. Moreover, skin dimples and epithelial inclusion cysts caused by percutaneous puncture of the suture needle are common occurrences, and extrusion of the suture knots can occur if the knots are not completely buried. Additionally, partial relapse occurs due to a decrease in the tensile strength of the suture or the cheese-cutting effect. Furthermore, we felt that it would be advantageous to perform this procedure in adult patients with hard auricular cartilage, because the procedure is currently indicated only for young patients with soft and pliable auricular cartilage.

In this article, we introduce our modifications and demonstrate the clinical usefulness of our method for correcting prominent ear in patients with hard auricular cartilage.

\section{METHODS}

The basic principle of our procedure is that traction sutures were placed from the scapha to the mastoid via percutaneous punctures without skin undermining and cartilage manipulation, as in PACO. The traction vector was perpendicular to the auricular cartilage,

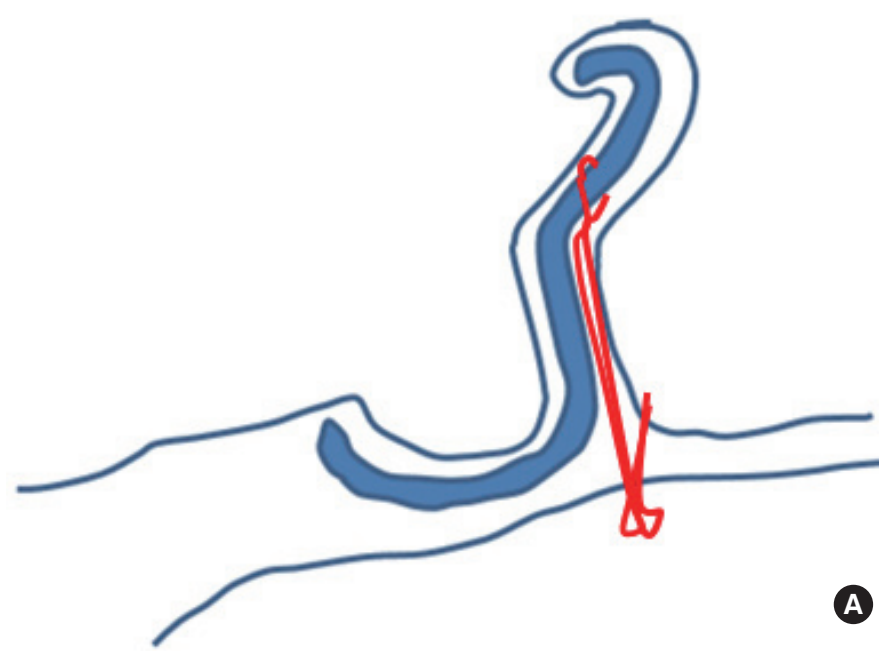

thereby creating an adequate conchoscaphal angle and auriculocephalic distance (Fig. 1). Operations were performed under local anesthesia. During intraoperative inspection, the auricle was folded into a normal anatomical shape to determine the traction site. Based on the anatomical features of the patients, three lines (upper, middle, and lower; each 7-8 $\mathrm{mm}$ in length) were drawn parallel to the antihelix on the scapha and counter scapha on the posterior surface of the auricle (Fig. 2A). A small amount of $2 \%$ lidocaine with 1:100,000 epinephrine was injected gently for bleeding control and focal hydrodissection with a 30 -gauge needle. Tab incisions were made with a \#11 scalpel at both ends of all lines to prevent skin dimples and epithelial inclusion cysts, which result from dragging the dermis and the epithelium into the subcutaneous suture tract. A cutting needle with a 4-0 nylon suture was passed from the counter scapha to the scapha through the lower hole of the middle line and reinserted into the exit point on the scapha. The suture was passed between the anterior perichondrium and skin, and drawn out through the upper hole of the middle line on the scapha. The suture was reinserted into the upper hole of the middle line on the scapha and drawn through the upper hole of the middle line on the counter scapha (Fig. 2A). The sutures in the upper and lower lines were placed in the same manner. At the posterior side of the auricle, six traction lines indicating the courses of the traction sutures were drawn from the suture exits on the counter scapha to the postauricular sulcus (Fig. 2B). A small amount of $2 \%$ lidocaine was gently injected on the posterior side of the auricle. Tab incisions were made at the postauricular sulcus to ensure that the knots were completely buried. An empty curved needle (18 $\mathrm{mm}$ in length) was threaded with the lower arm of the suture in the middle line. It was reinserted into the lower hole of the middle line on the counter scapha, passed between the posterior perichondrium and skin along

Fig. 1. The basic principle of percutaneous adjustable closed otoplasty. (A) Traction sutures are placed from the scapha to the mastoid via percutaneous punctures without skin undermining or cartilage manipulation. (B) After tightening of the knots; the traction vector is perpendicular to the auricular cartilage, thereby creating an adequate conchoscaphal angle and auriculocephalic distance. 

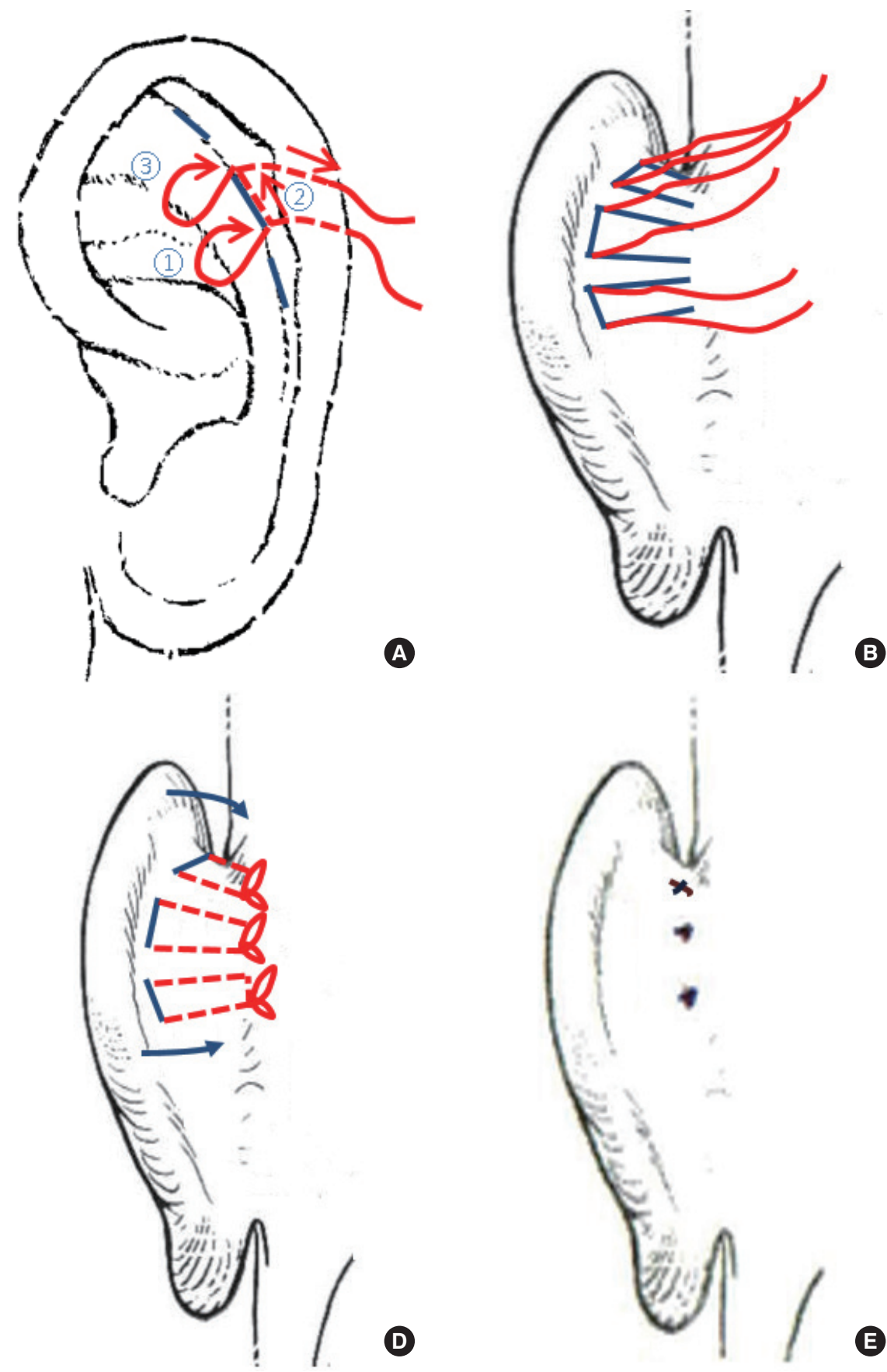

(D)

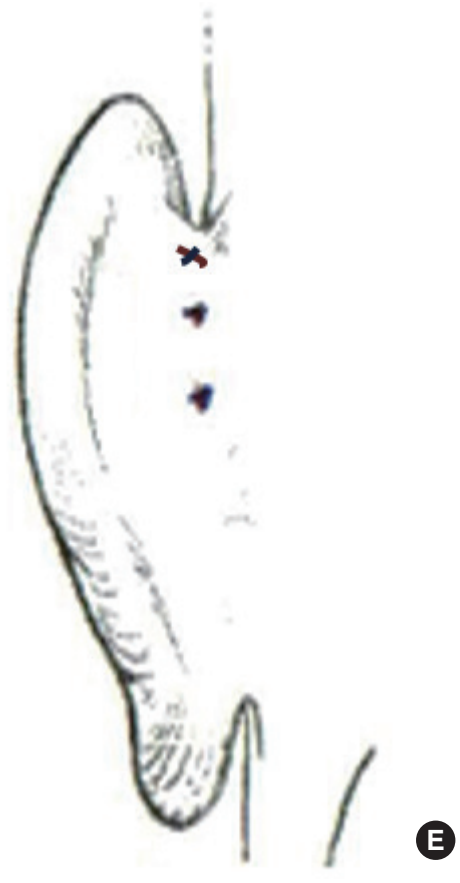

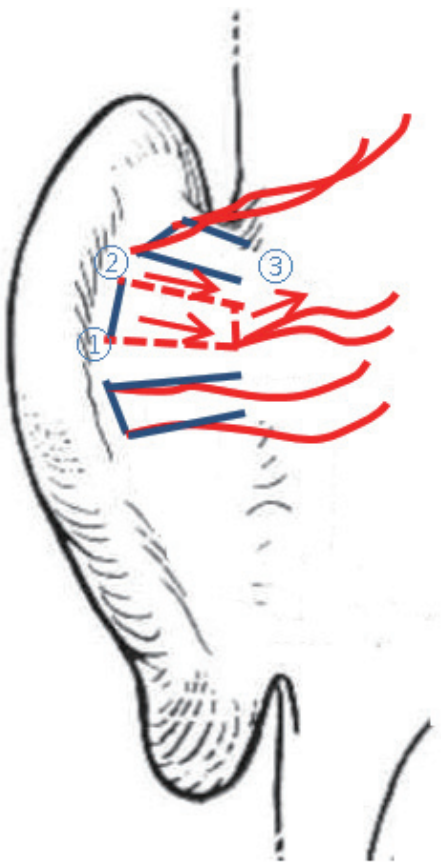

C

Fig. 2. The modified closed cartilagepreserving otoplasty technique. (A) Based on the anatomical characteristics of the patient, three lines (upper, middle, and lower; each 7-8 $\mathrm{mm}$ in length) were drawn parallel to the antihelix on the scapha and counter scapha on the posterior surface of the auricle. Tab incisions were made with a \#11 scalpel at both ends of all lines to prevent skin dimples and epithelial inclusion cysts, which result from dragging the dermis and the epithelium into the subcutaneous suture tract. A cutting needle with a 4-0 nylon suture was passed through the lower hole of the middle line from the counter scapha to the scapha and reinserted into the exit point. The suture was passed between the anterior perichondrium and skin, and out through the upper hole of the middle line. It was rein-

serted into the exit point and drawn out through the counter scapha. (B) The sutures at the upper and lower lines were placed in the same manner. On the posterior side of the auricle, six traction lines indicating the courses of the traction sutures were drawn from the suture exits on the counter scapha to the postauricular sulcus. (C) An empty curved needle (18 $\mathrm{mm}$ in length) was threaded following the lower arm of the suture in the middle line. It was reinserted into the lower hole of the middle line on the counter scapha, passed between the posterior perichondrium and skin along the traction line, and drawn out through the tab incision in the postauricular sulcus. The upper arm was delivered to the postauricular sulcus in the same manner, reinserted into its exit point in the postauricular sulcus, and delivered to the lower exit point by being scraped over the mastoid periosteum such that it was anchored to the mastoid periosteum. (D) The sutures of the upper and lower traction sites were placed in the same manner. After determining an adequate auriculocephalic distance, all knots were tied four times to prevent unravelling. A slight overcorrection was performed to compensate for post-surgical relapse. (E) The excess sutures were cut off. Tab incision openings were closed by one stitch with a 6-0 nylon suture. 
the traction line, and drawn out through the tab incision of the postauricular sulcus. The upper arm was delivered to the postauricular sulcus in the same manner. It was then reinserted into its exit point in the postauricular sulcus and delivered to the lower exit point by being scraped over the mastoid periosteum, thus anchoring it to the mastoid periosteum (Fig. 2C). The sutures of the upper and lower traction sites were placed in the same manner. After determining an adequate auriculocephalic distance, all knots were tied four times to prevent unravelling, beginning with the middle traction sites. A slight overcorrection was made to compensate for post-surgical relapse (Fig. 2D). Excess sutures were trimmed at the exit point. Tab incision openings where the knots were buried in the postauricular sulcus were closed with one stitch with a 6-0 nylon suture (Fig. 2E). Other tab incision sites were not closed. No drains were inserted.

Antibiotic ointment was applied over all incision sites and a sim-
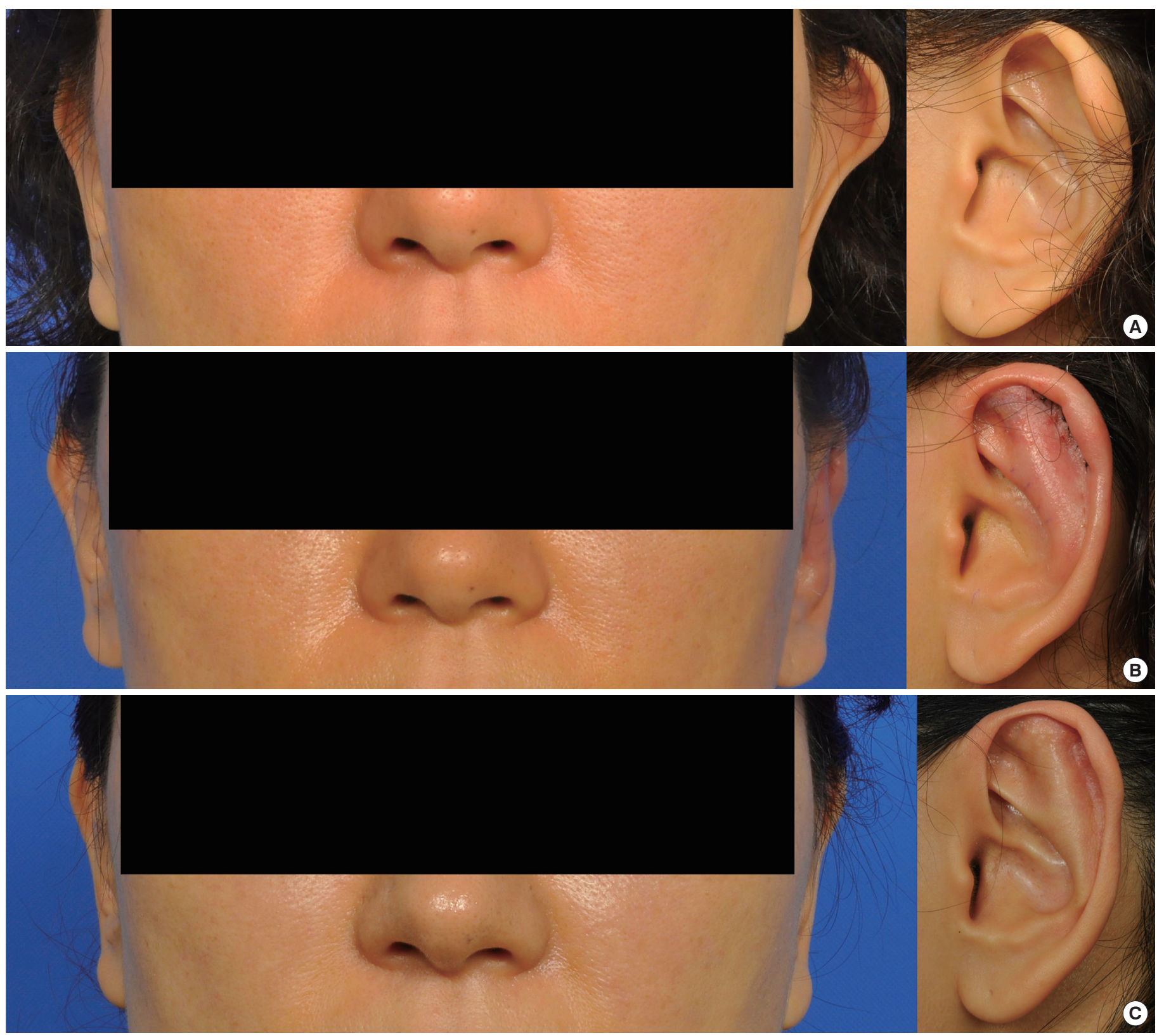

Fig. 3. Case 1. (A) Preoperative photographs of a 44-year-old woman with left prominent ear showing an absence of the antihelical fold. (B) Photographs taken two days postoperatively showing mild focal swelling without hematoma or skin dimples at the puncture sites. This patient simultaneously underwent correction for an underdeveloped helical roll using retention sutures and minor soft tissue excision. (C) Photographs taken eight months postoperatively showing a well-defined antihelical fold without contour deformity. 
ple moisturized gauze dressing was applied. No postoperative bandages were used. Patients returned to their normal lives without hospitalization. The patients were instructed not to rub or move the operated ears in a forward direction.

\section{RESULTS}

Two women (44 and 20 years of age, respectively) with a prominent upper half of the ear underwent this procedure under local anesthesia. The operations were performed in an outpatient setting, and operations lasted 45 minutes and 35 minutes, respectively. In one case, additional application of retention sutures and a minor soft tissue excision were used to simultaneously correct an underdeveloped helical roll. Minimal postoperative swelling lasted for seven days. We observed no complications such as hematoma, infection, chondritis, scarring, suture-related skin erosion, epithelial inclusion cyst formation, suture extrusion, suture breakage, contour deformities, or dimples on the scapha. At six and eight months postoperatively, minimal relapse (2-3 $\mathrm{mm}$, respectively) occurred, but this was compensated for by overcorrection during the surgery. Both patients were left with well-defined antihelical folds and were satisfied with the aesthetic results (Fig. 3 and 4). In one case, a pal- pable and slightly visible suture band was observed on the postauricular surface, but the patient did not complain of this and it did not require a secondary procedure. Excessive postauricular skin that appeared in the early postoperative period spontaneously resolved during the follow-up period (Fig. 5).

\section{DISCUSSION}

More than 200 techniques have been suggested for the surgical correction of prominent ear [1]. No consensus exists regarding the best technique, and novel techniques and modifications therefore continue to be developed. Otoplasty techniques can be divided into three broad categories: cartilage-cutting techniques, cartilage-sparing techniques, and incisionless techniques [5]. Cartilage-sparing techniques may involve scoring of the cartilage, but they are referred to as cartilage-sparing because no complete cartilaginous incisions are made [8]. We categorized otoplasty techniques more specifically to avoid confusion. The surgical techniques for prominent ear correction include two procedures: (1) skin incision and undermining, and (2) cartilage manipulation. These procedures may be open or closed, and differ regarding the use of skin incisions and undermining. Cartilage-cutting, cartilage-scoring, and
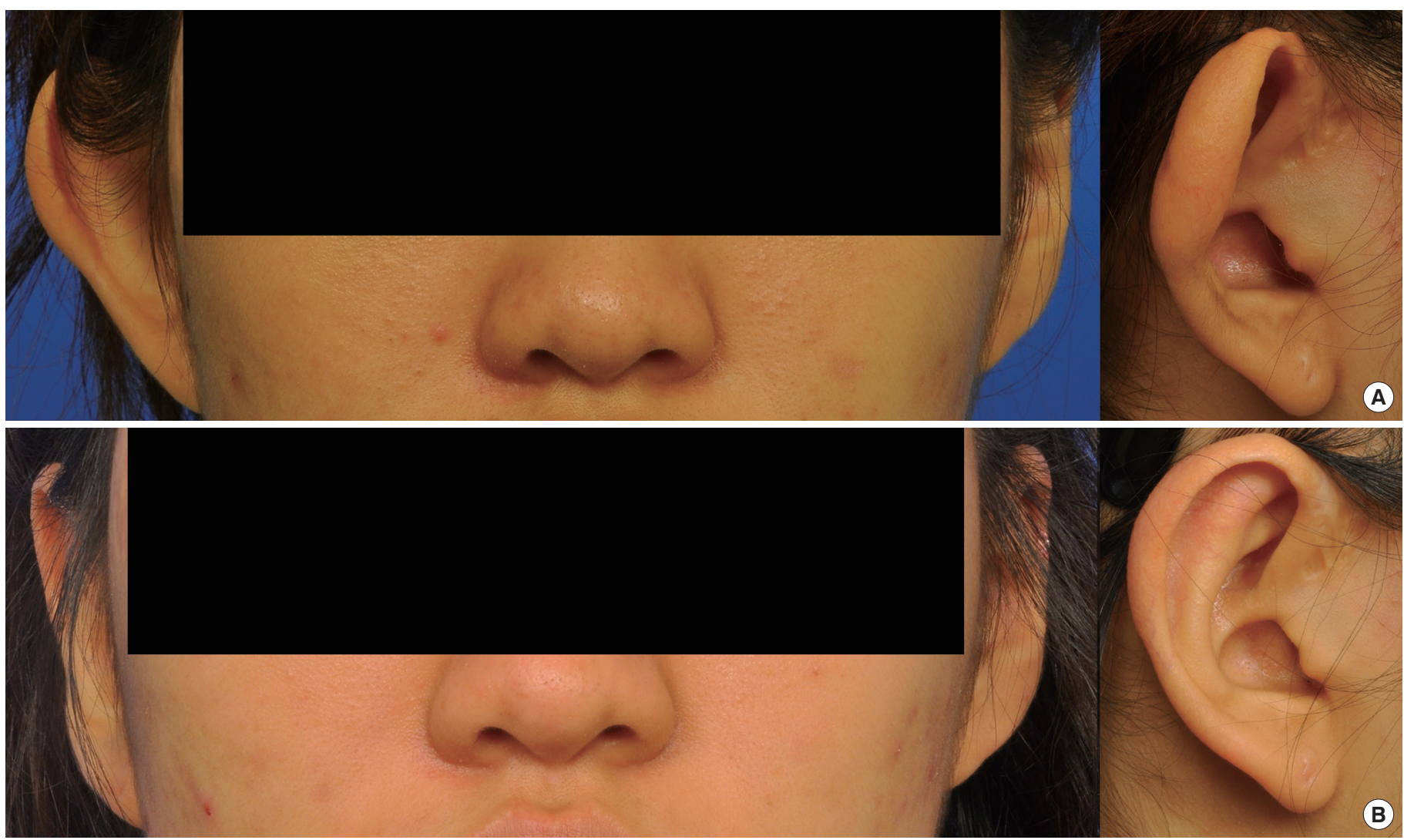

Fig. 4. Case 2. (A) Preoperative photographs of a 20 -year-old woman with right prominent ear showing an absence of an antihelical fold. (B) Photographs showing taken six months postoperatively showing a well-defined antihelical fold without contour deformity. 

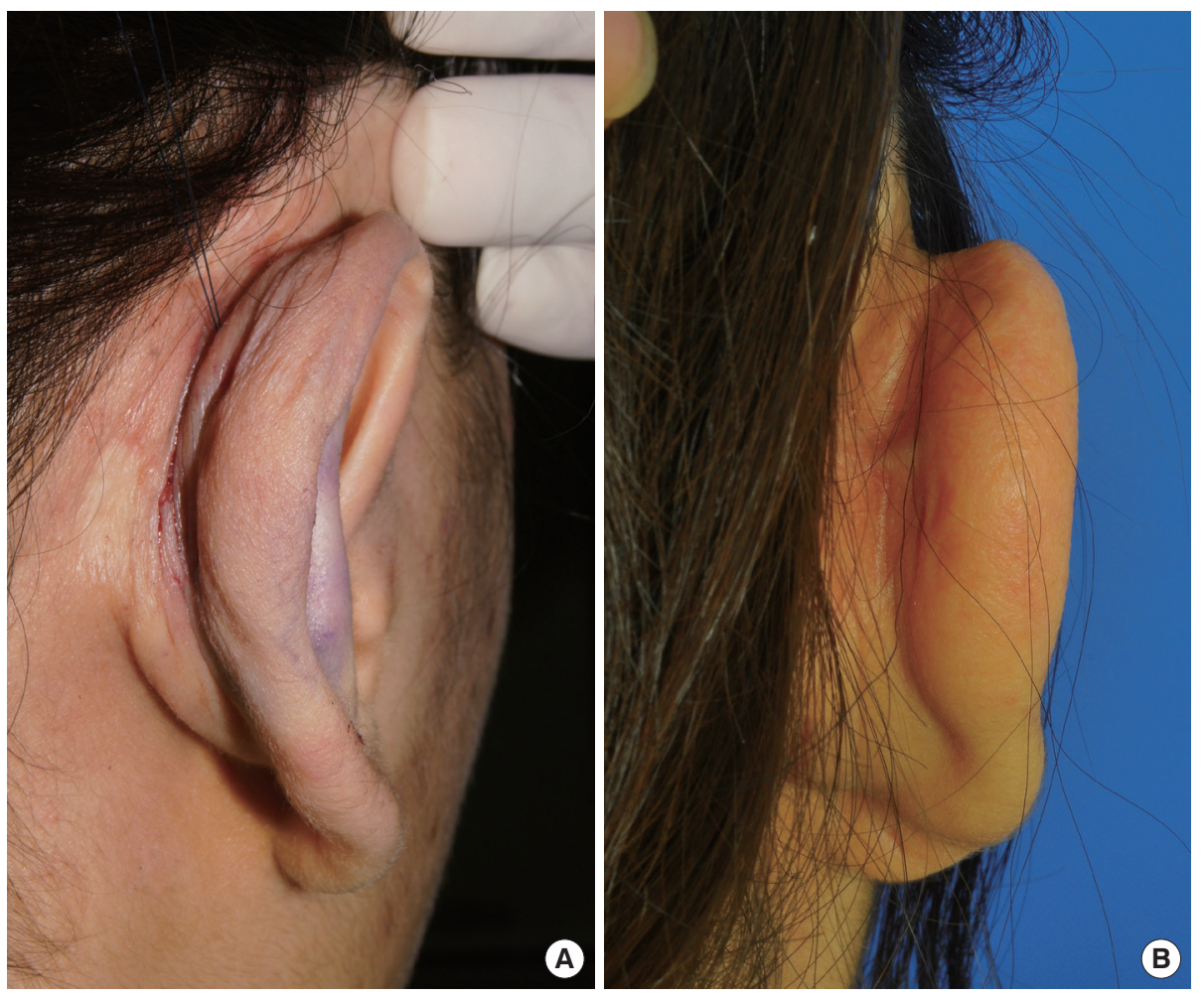

Fig. 5. Excessive postauricular skin. (A) Intraoperative photograph showing excessive postauricular skin created by a change in the shape of the ear. (B) Photograph taken six months postoperatively showing spontaneous resolution of the excessive postauricular skin. cartilage-preserving procedures differ according to the use of cartilage manipulation. The surgical techniques for prominent ear correction combine these two principles.

Cartilage-scoring otoplasty has been widely used for prominent ear correction since its concept was first introduced by Gibson and Davis [9] in 1955. In this approach, cartilage resistance is reduced by means of scoring or rasping, which facilitates formation of the antihelical fold and suturing of the cartilage. This approach also has the advantage of maximally preserving cartilage support and minimizing the emergence of contour disorders, as no full-thickness cartilaginous incisions are made $[5,6,8]$. Although minimalaccess scoring techniques have been developed $[10,11]$, such techniques require skin incision and undermining in order to score or rasp the cartilage. Therefore, they still pose the risk of hematoma and require postoperative drains or a compressive dressing $[5,6]$.

In 2009, Fritsch [7] first developed a closed cartilage-preserving otoplasty technique known as the incisionless otoplasty. This technique does not require skin incision, undermining, or cartilage manipulation; it only uses antihelix-forming sutures. Retention sutures from the scapha to the concha or from the concha to the mastoid are placed according to the patient's pathologic condition via percutaneous punctures. The traction vector of these sutures is oblique to the auricular cartilage. Fritsch stated that because it does not involve skin incisions, undermining, or cartilage manipulation, this technique poses no risk of hematoma, infection, disordered contours, or sharp edges, and the operation takes little time [7]. Compressive dressings or drains are not needed, as it involves no risk of hematoma. This procedure can be performed in an outpatient setting because it is associated with a short operation time, minimal pain, and no risk of complications [7].

In 2013, Ozturan et al. [5] refined this technique and developed a novel incisionless technique known as PACO. In PACO, three traction sutures are placed from the scapha to the mastoid via percutaneous punctures. The traction vector is perpendicular to the auricular cartilage. In addition, the auriculocephalic distance can be adjusted while tightening the suture knots and monitoring the distance from the auricle to the scalp with a ruler. The researchers proposed that PACO may not be an effective treatment for ears with thick and sturdy cartilage, and therefore performed PACO only on ears with soft cartilage. In their study, PACO showed rates of efficacy, recurrence, complications, and patient satisfaction comparable to those of the cartilage-scoring techniques that are currently in wide use [6]. They concluded that PACO is preferable to cartilage-scoring techniques for the correction of prominent ear in ears with soft cartilage [6].

The PACO technique is very effective and has many advantages; however, we introduced certain modifications in order to make the procedure more practical and accessible and to achieve better results, and also demonstrated the utility of this modified technique in adult patients with hard auricular cartilage.

First, we used a 4-0 nylon suture to resolve problems related to the suture material. In previous closed cartilage-preserving otoplasty techniques, 3-0 braided white polyester sutures were used because they are strong, soft, easily handled, and invisible. Howev- 
er, 3-0 braided white polyester sutures are the most costly sutures available and their high coefficients of friction can cause them to pass through the tissues with considerable drag, which can result in formation of epithelial inclusion cysts [12] and skin dimples. Furthermore, due to their braided structure, partial breakage can occur if the ear is accidentally moved forward [7]. 4-0 nylon sutures are thinner, but have high tensile strength. As they are monofilament sutures, the coefficient of friction is low and partial breakage does not occur. In addition, they have minimal tissue reactivity, are affordable, and are easy to obtain in most medical institutions. The possible unravelling of a knot given the high degree of memory exhibited by nylon can be resolved by tying it more times. Although these sutures are blue, they are not visible because they are subcutaneous and close to the auricular cartilage. Previous reports have indicated that after years of having been in a certain position, auricular cartilage and soft tissues may retain a certain shape $[7,13]$. Nylon hydrolyzes very slowly, so hydrolysis occurring years after the operation is unlikely to be a problem.

Second, tab incisions were made at all puncture sites. On the scapha, we made tab incisions at predetermined puncture sites to avoid the formation of skin dimples or epithelial inclusion cysts that may be caused by dragging of the dermis or the epidermis into the subcutaneous suture tracts. We made tab incisions on the postauricular sulcus to ensure that the suture knots could be completed in a buried manner. Suture extrusion is a common complication of otoplasty. The rates of suture extrusion in cartilage-scoring techniques and in the PACO technique have been found to be $12.5 \%$ and $17.8 \%$, respectively [6]. No suture extrusions occurred in any knot in our cases.

Third, an empty curved needle (18 $\mathrm{mm}$ in length) was used as a suture carrier to manipulate the sutures on the postauricular sulcus. For PACO, the surgeons used a specially designed suture carrier $[5,6]$. The use of an empty curved needle makes our procedure more practical. Empty curved needles are easily obtainable in most operating rooms and their degree of curvature allows them to be passed along the curved postauricular surface under the postauricular skin.

Fourth, slight overcorrection was performed. The auriculocephalic distance was augmented by $20 \%$ in comparison with the preoperative values in both the cartilage-scoring techniques and in the PACO technique [6]. Therefore, we employed a slight overcorrection to compensate for post-surgical relapse caused by a decrease in the tensile strength of the nylon or the cheese-cutting effect.

Fifth, we applied this procedure to adult patients with hard auricular cartilage, whereas PACO is indicated only for young patients with soft and pliable auricular cartilage. We took into account that the perpendicular traction vector of the retention sutures used in PACO delivers the traction force more efficiently than does the oblique traction vector of the retention sutures used in incisionless otoplasty techniques and the Mustarde cartilage scoring technique.

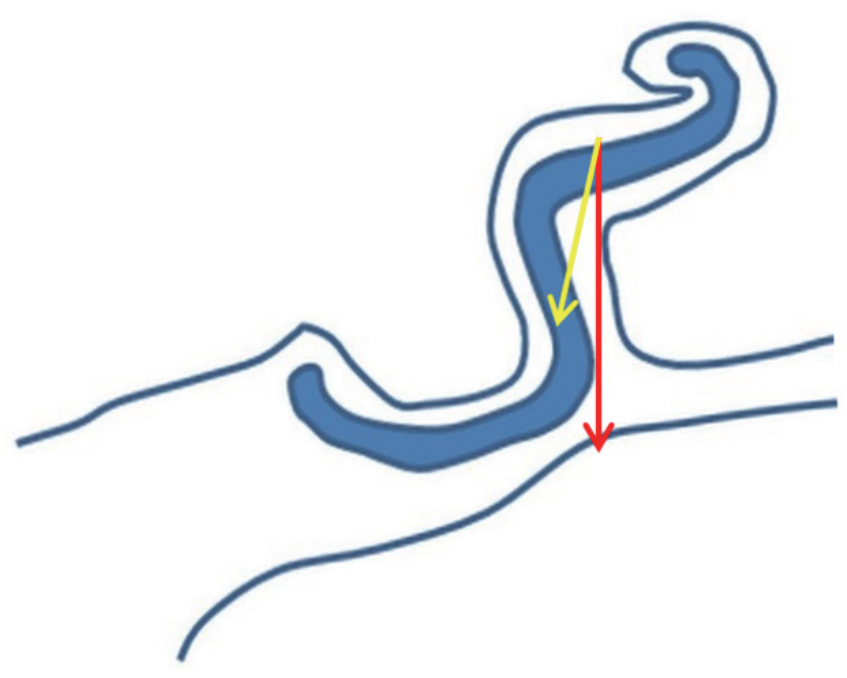

Fig. 6. The traction vector of the retention sutures. The perpendicular traction vector (red arrow) to the scapha used in the percutaneous adjustable closed otoplasty (PACO) technique distributes the traction force more efficiently than does the oblique traction vector lyellow arrow). The oblique traction vector requires a greater force in order to create a force equal to that of the perpendicular traction vector.

Oblique traction vectors require a greater force in order to equal that of a perpendicular traction vector (Fig. 6), and this difference may result in a cheese-cutting effect on the cartilage. Thus, we reasoned that when a perpendicular traction vector was used, the corrected state would be well-maintained without the cheese-cutting effect, even in adult cartilage. We saw satisfactory results in longterm follow-up examinations.

\section{CONCLUSIONS}

In our modified closed cartilage-preserving otoplasty technique, the suture material and suture carrier were changed to inexpensive and more common materials that can be easily obtained in most operating rooms. Tab incisions were created to prevent skin dimples and epithelial inclusion cysts and to completely bury the suture knots. Slight overcorrection was performed to compensate for relapse. Consequently, we achieved satisfactory results in adult patients with hard auricular cartilage without the problems associated with PACO. Given the results of our study, we propose that our technique may be a reliable treatment option for prominent ear correction.

The limitations in this study are that it included a small number of cases and did not include a comparison of our results with those obtained using other otoplasty techniques. Additional cases with long-term follow up and a comparative case study with other otoplasty techniques will be necessary. 


\section{PATIENT CONSENT}

Patients provided written consent for the use of their images.

\section{REFERENCES}

1. Adamson PA, Strecker HD. Otoplasty techniques. Facial Plast Surg 1995;11:284-300.

2. Brent BD. Reconstruction of the auricle. In: Mathes SJ, Hentz VR, editors. Plastic surgery. 2nd ed. Philadelphia, PA: Saunders; 2006. p.63398.

3. Bradbury ET, Hewison J, Timmons MJ. Psychological and social outcome of prominent ear correction in children. Br J Plast Surg 1992;45: 97-100.

4. LaTrenta GS. Otoplasty. In: Rees TD, LaTrenta GS, editors. Aesthetic Plast Surg. 2nd ed. Philadelphia, PA: Saunders; 1994. p.891-924.

5. Ozturan O, Dogan R, Eren SB, et al. Percutaneous adjustable closed otoplasty for prominent ear deformity. J Craniofac Surg 2013;24:398404.
6. Ozturan O, Dogan R, Eren SB, et al. Cartilage-sparing techniques versus percutaneous adjustable closed otoplasty for prominent ear deformity. J Craniofac Surg 2014;25:752-7.

7. Fritsch MH. Incisionless otoplasty. Otolaryngol Clin North Am 2009; 42:1199-208, Table of Contents.

8. Petersson RS, Friedman O. Current trends in otoplasty. Curr Opin Otolaryngol Head Neck Surg 2008;16:352-8.

9. Gibson T, Davis WB. Some further observations on the use of preserved animal cartilage. Br J Plast Surg 1955;8:85-92.

10. Thomas SS, Fatah F. Closed anterior scoring for prominent-ear correction revisited. Br J Plast Surg 2001;54:581-7.

11. Pereira-Filho O, Eickhoff DR, Bins-Ely J, et al. Novel approach to closed treatment of prominent ear without skin resection (endotoplasty). Plast Reconstr Surg 2006;118:136-8.

12. Macht SD, Krizek TJ. Sutures and suturing--current concepts. J Oral Surg 1978;36:710-2.

13. Matsuo K, Hayashi R, Kiyono M, et al. Nonsurgical correction of congenital auricular deformities. Clin Plast Surg 1990;17:383-95. 